\title{
A Greedy Double Auction Mechanism for Grid Resource Allocation
}

\author{
Ding Ding ${ }^{\star \star}$, Siwei Luo, and Zhan Gao \\ Beijing Jiaotong University, Beijing 100044, China
}

\begin{abstract}
To improve the resource utilization and satisfy more users, a Greedy Double Auction Mechanism(GDAM) is proposed to allocate resources in grid environments. GDAM trades resources at discriminatory price instead of uniform price, reflecting the variance in requirements for profits and quantities. Moreover, GDAM applies different auction rules to different cases, over-demand, over-supply and equilibrium of demand and supply. As a new mechanism for grid resource allocation, GDAM is proved to be strategy-proof, economically efficient, weakly budgetbalanced and individual rational. Simulation results also confirm that GDAM outperforms the traditional one on both the total trade amount and the user satisfaction percentage, specially as more users are involved in the auction market.
\end{abstract}

\section{Introduction}

Due to the specialities such as geographical distribution, heterogeneity and site autonomy, it is hard and challenging to manage grid resources. Fortunately, with the emergence of the grid economy, economic-based model is proposed to allocate resources in grid. This economic mechanism includes auctions, commodity markets, tenders and posted price and has many attractive features[1,2].

Auction-based resource allocation has attracted much attentions since it requires less global information, has decentralized structure and is easy to implement. Depending on the type of interactions between sellers and buyers, auctions can be classified into two classes, one-sided auctions and two-sided auctions. In one-sided auctions, only grid users submit bids to a central auctioneer, while in two-sided auctions, also called double auctions, both users and resource owners submit bids. The selling price and the users and the resource owners that trade are decided by the central auctioneer based on different types of double auction mechanisms. According to the trading units, the double auction can be classified as SDA (Single-unit Double Auction), where at most one unit of resource can be traded in one auction, and MDA (Multi-unit Double Auction), where more than one unit of resource can be traded in one auction. MDA is more suitable for a huge number of buyers and sellers trading through network, thus can be well applied to resource allocation in a grid environment.

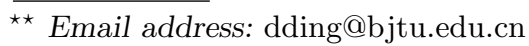


The earliest work on economic-based resource allocation can be traced back to 1968 when Sutherland proposed the auction mechanism for resource allocation in PDP-1 machine[3]. GRACE [4] presented a economic-based grid resource management architecture and described several economic models. Popcorn [5] was a Web based computing market, which adopted both one-sided and twosided auctions to realize on-line resource allocation across the Internet. Spawn [6] managed heterogeneous computer resources based on Vickery auction, so did CORE [7]. Double auctions were used in JaWS [8] and preferred by many projects such as Tycoon [9]. However, these projects only adopted the basic auction methods instead of researching them deeply. In [10] and [11], a SDA market is designed, but only the strategy behavior on the buyers side was considered. Still in a SDA, [12] considered strategy behaviors both on the sellers and buyers sides. In [13], a MDA mechanism was proposed for the electric market, using the uniform auction. Different double auction protocols are compared in [14] and combinatorial MDA for resource allocation was studied in [15] and [16]. Double auction is also further used in grid scheduling $[17,18]$.

In this paper, we propose a double auction based resource allocation mechanism, a greedy MDA, trying to make the resource consumers and provides trade as more as possible under the guarantee of their QoS demands(that is the requirements for profits and quantities).

The rest of this paper is organized as follows. The next section gives the basic framework of the auction market. In section 3 the disadvantages of the traditional auction mechanism are described and GDAM is presented. Some features of GDAM are proved in section 4. In section 5, simulation experiments are conducted and the results are discussed. The last section includes the conclusion of this paper.

\section{The Basic Market Framework}

Generally, entities that can be traded in a grid market are different kinds of grid resources, such as storage resource and computing resource. In this paper we focus on auctions for only one kind of grid resource and the combinatorial auction is not considered. The auction market is constituted by three components, Seller Agent (SA), who works on behalf of sellers, Buyer Agent (BA), who works on behalf of buyers and Auction Agent (AA), who manages the auction market. A buyer will send to its BA the buy request, $b r=(q, p)$, where $b r . q$ is the quantity of the resource it needs and br.p is the bidding price for a unit of the resource. A seller will send to its SA the sell request, $s r=(q, p)$, where $s r . q$ is the quantity of the resource it wants to sell and $s r . p$ is the asking price for a unit of the resource. And neither a SA nor a BA knows the treading requests of other agents. After receiving the trading requests from SAs and BAs, AA makes use of a certain double auction mechanism to decide the buyers and seller who can trade and the amounts and prices of the resources that will be traded. AA organizes double auctions at regular time interval, and BAs and SAs submit their own trading requests at the auction period. 
The market framework is illustrated in figure 1.

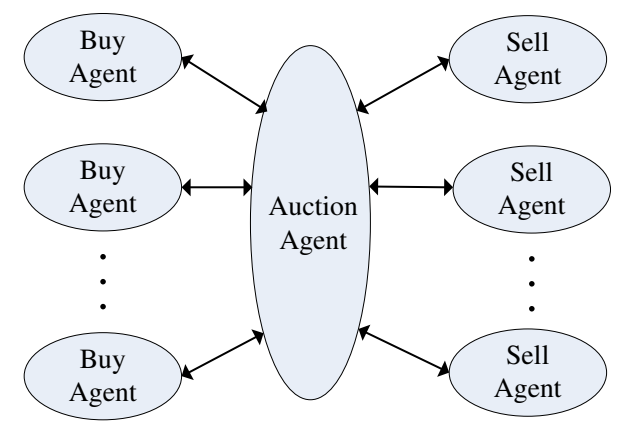

Fig. 1. The basic market framework.

\section{Greedy Double Auction Mechanism}

Most researches on double auction in the economic field aim at maximizing the total market value, that is, maximizing the collective surplus of the market participants. However, these mechanisms are not suitable for the grid. Firstly, before the final trading price is determined by AA, neither SAs nor BAs know how much profit they will get from trading per unit of resource. Thus, the market can not provide a definite profit guarantee for the participants. Secondly, under traditional mechanisms the final trading price for all the grid resources is the same, which is unfair. A resource with a better quality may have a higher cost, and is supposed to be traded at a higher price. Thirdly, in order to maximize the collective surpluses, traditional mechanisms tend to find the buyers' and sellers' reservation prices between which there are the largest gaps to make them trade. This causes two problems. On one hand, the market value is shared by the minority and is distributed unbalanced for only a small percentage of participants can successfully trade, especially when the number of participants is large. On the other hand, the utilization of grid resources is low. Few buyers can eventually benefit from the resources they need, making many resources, which can potentially be traded and utilized, idle. In this paper, a greedy double auction mechanism(GDAM) is proposed which does not focus on how to maximize the total market value but how to improve the resources utilization and benefit the majority of the market participants.

Under GDAM, the auction market provides trading service for the participants, using trading price and trading amount as two QoS parameters. As for a seller $j$ he requires the selling service with $s r_{j} . p$ and $s r_{j} . q$ as his QoS requirements; as for a buyer $i$ he requires the buying service with $b r_{i} . p$ and $b r_{i} . q$ as his QoS requirements. A seller's asking price is his expected profit plus the cost 
of providing per unit of resource, while a buyer gets his bidding price by subtracting the cost from the value created by consuming per unit of the resource. Let seller $j$ 's cost of providing per unit of resource be $s c_{j}$ and the value created by buyer $i$ 's consuming per unit of resource be $c v_{i}$. Let $q_{i j}$ denote the quantity buyer $i$ buys from seller $j$, then the surplus of buyer $i$ for this transaction is

$$
s b_{i}=\left(c v_{i}-b r_{i} \cdot p\right) b r_{i} \cdot q,
$$

and the surplus of seller $j$ is

$$
s s_{j}=\left(s r_{j} \cdot p-s c_{j}\right) s r_{j} \cdot q .
$$

By dong this, the profits of the successful participants can be guaranteed. With trading at the expected price, no agents will complain about the unfairness. And our mechanism works in three different cases.

Case I: Supply over Demand (SoD) In this case, there are more supply quantities than demand quantities available in the auction market. Let $m$ be the number of buyers and $n$ be the number of sellers then (3) holds.

$$
\sum_{j=1}^{n} s r_{j} \cdot q \geq \sum_{i=1}^{m} b r_{i} \cdot q+T(T>0)
$$

Without loss of generality, we assume

$$
\begin{aligned}
& b r_{1} \cdot p \geq b r_{2} \cdot p \ldots \geq b r_{m} \cdot p, \\
& s r_{1} \cdot p \geq s r_{2} \cdot p \ldots \geq s r_{n} \cdot p .
\end{aligned}
$$

According to (4) and (5), GDAM arranges the demand quantities and supply quantities in the descendent order of price(refer to figure 2). We can see from figure 2 that the price-quantity broken line of the buyers crosses that of the sellers at some crossing points. These crossing points can be divided into two classes: up-crossing point $f_{i}$ and down-crossing point $g_{i} . f_{i}$ occurs when $(6)$ and (7) hold, and we call $b r_{K}$ and $s r_{L}$ up critical requests.

$$
\begin{gathered}
b r_{K+1} \cdot p<s r_{L} \cdot p<b r_{K} \cdot p \\
\sum_{i=1}^{L} s r_{i} \cdot q>\sum_{i=1}^{K} b r_{i} \cdot q
\end{gathered}
$$

If and only if $f_{i}$ exists and (8) and (9) hold, $g_{i}$ will be generated, and we call $s r_{F}$ and $b r_{E}$ down critical requests.

$$
\begin{gathered}
s r_{F+1} \cdot p<b r_{E} \cdot p<s r_{F} \cdot p \\
\sum_{i=1}^{E} b r_{i} \cdot q>\sum_{i=1}^{F} s r_{i} \cdot q
\end{gathered}
$$




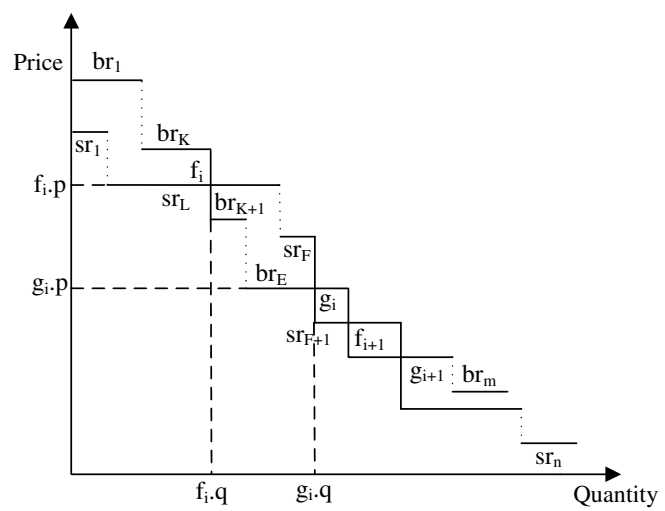

Fig. 2. Supply over Demand.

With all the trading requests submitted to AA by SAs and BAs, AA will use the SoD auction rule illustrated in figure 3 .

In step (2) and (3), we remove the the selling requests with too high asking prices and rank all the left requests, including demand and supply, in the decreasing order of price. During the next do loop(step (4) to (8)), the first up-crossing point and down-crossing point, along with the corresponding critical requests $S_{L}$ and $b r_{E}$, is found, and then the leading selling request $S_{i}$ whose asking price is under bidding price of $b r_{E}$ are figured out. To satisfy more buying requests, we replace the critical selling request $S_{L}$ with the new selling request $S_{i}$ by shifting the position of $S_{i}$ to that of $S_{L}$. It is motivated by the fact that there are more supply quantities than demand quantities and the buying requests have a greater impact on the total trade quantities.

The do loop ends under two conditions. One is that there are no more upcrossing points, which means the entire price-quantity broken line of sellers is below that of buyers. The other is that there is only a up-crossing point but no down-crossing points, which indicates that the price-quantity broken line of buyers is above that of sellers before the up-crossing point while the pricequantity broken line of buyers is below that of sellers after the up-crossing point. The first condition is processed from step (9) to (19). In the first if-else statement(step (10) to (16)), the selling request $S_{S N}$, and buying request $b r_{B N}$, with the smallest price margin between each other, are figured out. After that, buying requests $b r_{i}$ with indices $i<B N$ and sellers with indices $j<S N$ participate in trade(step (17) to (19)). Note that $S_{S N}$ and $b r_{B N}$ are sacrificed in order to avoid the partial trade which couldn't meet the QoS needs of trading quantity. Though step (18) and step (19) can also lead to partial trade, this cut of a buyer's demand quantity or a seller's supply quantity is usually so small that it can be neglected without degrading the QoS of auction market. In step (18), if $S_{j} . q<\sum_{j=1}^{S N-1} S_{j} . q-\sum_{i=1}^{B N-1} b r_{i} \cdot q /(S N-1)$, seller $j$ sells nothing and the 


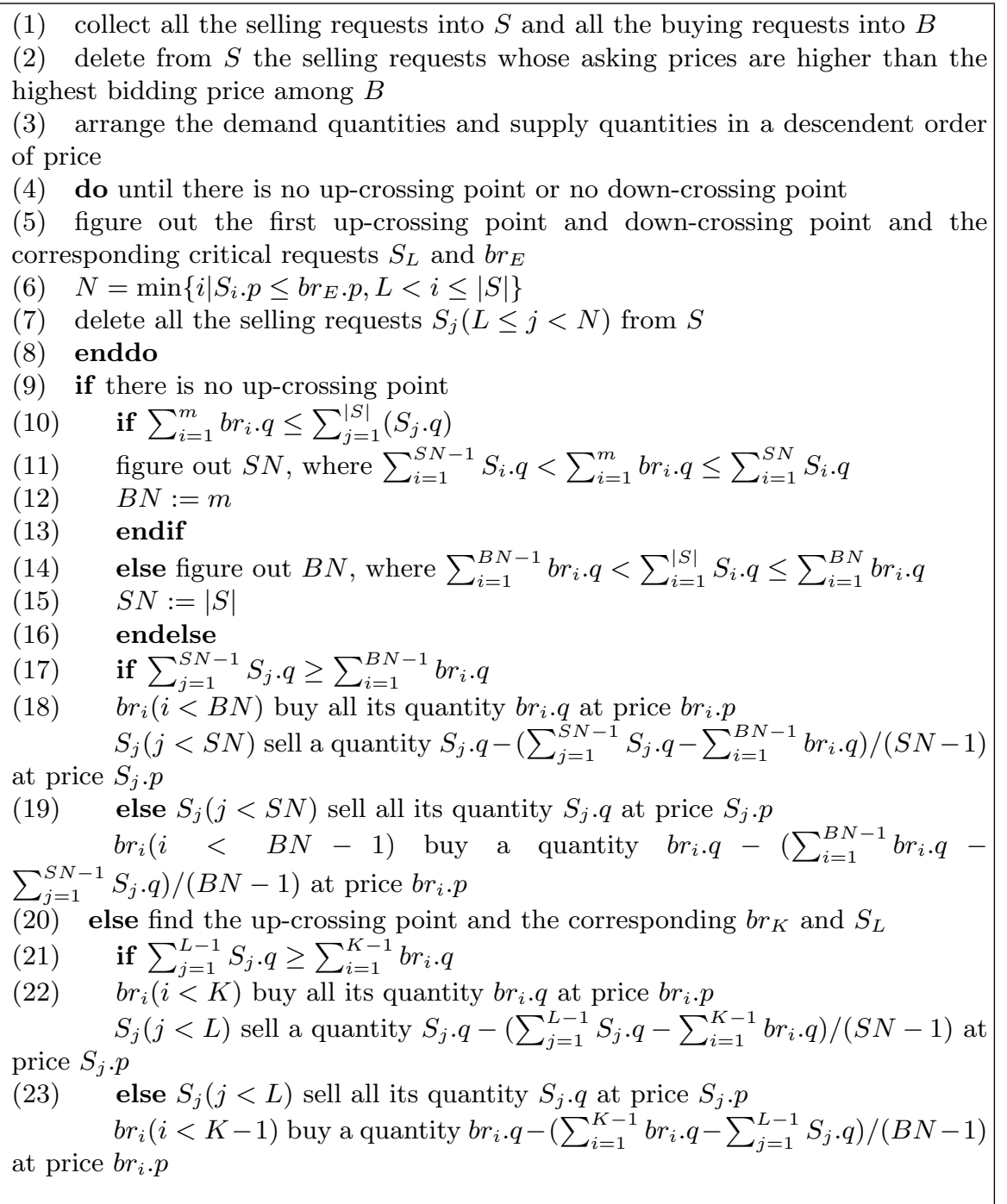

Fig. 3. The SoD auction rule in case of supply over demand.

"burden", $\sum_{j=1}^{S N-1} S_{j} \cdot q-\sum_{i=1}^{B N-1} b r_{i} \cdot q /(S N-1)-S_{j} . q$, will be split equally by the rest of $S N-2$ sellers. The procedure keeps running until each remaining seller trades a positive quantity. Step (19) involves the same situation of buyers. The second condition is handled in the last four steps(step (20) to (23)). Only $\mathrm{K}$ buyers and $\mathrm{L}$ sellers before the up-crossing point can trade and the processing method is similar to the first one. 
Case II: Demand over Supply (DoS) In this case, there are more demand quantities than supply quantities available in the auction market. Then (10) holds.

$$
\sum_{j=1}^{n} s r_{j} . q+T<\sum_{i=1}^{m} b r_{i} . q(T>0)
$$

Without loss of generality, we assume

$$
\begin{aligned}
& b r_{1} \cdot p \leq b r_{2} \cdot p \ldots \leq b r_{m} \cdot p, \\
& s r_{1} \cdot p \leq s r_{2} \cdot p \ldots \leq s r_{n} \cdot p .
\end{aligned}
$$

This time, a DoS auction rule will be adopted by GDAM. The DoS rule is similar to the SoD rule in Case I except that the buying requests will be shifted to satisfy more selling requests since the selling requests have a greater impact on the total trade quantities and should be satisfied first. According to the DoS auction rule, GDAM arranges the demand quantities and supply quantities in the ascendent order of price(refer to figure 4 ). We can see from figure 4 , the price-

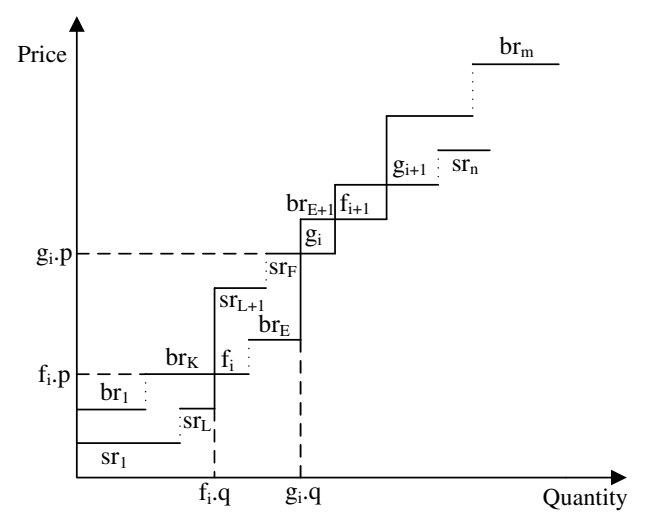

Fig. 4. Demand over Supply.

quantity broken line of the buyers crosses that of the sellers at some crossing points. The up-crossing point $f_{i}$ occurs when (13) and (14) hold, and we call $b r_{K}$ and $s r_{L}$ up critical requests.

$$
\begin{gathered}
s r_{L} \cdot p<b r_{K} \cdot p<s r_{L+1} \cdot p \\
\sum_{i=1}^{K} b r_{i} \cdot q>\sum_{i=1}^{L} s r_{i} \cdot q
\end{gathered}
$$

If and only if $f_{i}$ exsits and (15) and (16) hold, $g_{i}$ will be generated, and we call $s r_{F}$ and $b r_{F}$ down critical requests.

$$
b r_{E} \cdot p<s r_{F} \cdot p<b r_{E+1} \cdot p
$$




$$
\sum_{i=1}^{F} s r_{i} . q>\sum_{i=1}^{E} b r_{i} . q
$$

The DoS auction rule is shown in figure 5 .

(1) collect all the selling requests into $S$ and all the buying requests into $B$ (2) delete from $S$ the selling requests whose asking prices are higher than the highest bidding price among $B$

(3) arrange the demand quantities and supply quantities in the ascendent order of price

(4) do until there is no up-crossing point or no down-crossing point

(5) figure out the first up-crossing point and down-crossing point and the corresponding critical requests $B_{K}$ and $s r_{F}$

(6) $\quad N=\min \left\{i\left|B_{i} \cdot p \geq s r_{F} \cdot p, K<i \leq\right| B \mid\right\}$

(7) delete all the buying requests $B_{j}(K \leq j<N)$ from $B$

(8) enddo

(9) if there is no up-crossing point

(10) if $\sum_{i=1}^{|B|} B_{i} . q \leq \sum_{j=1}^{n}\left(s r_{j} . q\right)$

(11) figure out $S N$, where $\sum_{i=1}^{S N-1} s r_{i} . q<\sum_{i=1}^{|B|} B_{i} . q \leq \sum_{i=1}^{S N} s r_{i} . q$

(12) $\quad B N:=|B|$

(13) endif

(14) else figure out $B N$, where $\sum_{i=1}^{B N-1} B_{i} \cdot q<\sum_{i=1}^{n} s r_{i} . q \leq \sum_{i=1}^{B N} B_{i} . q$

(15) $\quad S N:=n$

(16) endelse

(17) if $\sum_{j=1}^{S N-1} s r_{j} . q \geq \sum_{i=1}^{B N-1} B_{i} \cdot q$

(18) $\quad B_{i}(i<B N)$ buy all its quantity $B_{i} . q$ at price $B_{i} . p$

$s r_{j}(j<S N)$ sell a quantity $s r_{j} \cdot q-\left(\sum_{j=1}^{S N-1} s r_{j} \cdot q-\sum_{i=1}^{B N-1} B_{i} \cdot q\right) /(S N-$

1) at price $s r_{j} \cdot p$

(19) else $s r_{j}(j<S N)$ sell all its quantity $s r_{j} . q$ at price $s r_{j} . p$ $B_{i}(i<B N-1)$ buy a quantity $B_{i} \cdot q-\left(\sum_{i=1}^{B N-1} B_{i} . q-\right.$ $\left.\sum_{j=1}^{S N-1} s r_{j} . q\right) /(B N-1)$ at price $B_{i} . p$

(20) else find the up-crossing point and the corresponding up critical requests $B_{K}$ and $s r_{L}$

(21) if $\sum_{j=1}^{L-1} s r_{j} \cdot q \geq \sum_{i=1}^{K-1} B_{i} \cdot q$

(22) $\quad B_{i}(i<K)$ buy all its quantity $B_{i} \cdot q$ at price $B_{i} . p$ at price $s r_{j} . p$

$S_{j}(j<L)$ sell a quantity $s r_{j} . q-\left(\sum_{j=1}^{L-1} s r_{j} . q-\sum_{i=1}^{K-1} B_{i} . q\right) /(S N-1)$

(23) $\quad$ else $s r_{j}(j<L)$ sell all its quantity $s r_{j} . q$ at price $s r_{j} . p$

at price $B_{i} \cdot p$

$B_{i}(i<K-1)$ buy a quantity $B_{i} \cdot q-\left(\sum_{i=1}^{K-1} B_{i} \cdot q-\sum_{j=1}^{L-1} s r_{j} \cdot q\right) /(B N-1)$

Fig. 5. The auction rule in case of demand over supply. 
Case III: Supply equals Demand In this case, the overall supply quantities are equivalent or nearly equivalent to the overall demand quantities. And (17) holds.

$$
\left|\sum_{j=1}^{n} s r_{j} . q-\sum_{i=1}^{m} b r_{i} \cdot q\right|<T(T>0)
$$

Our mechanism is very simple. The SoD rule in Case $\mathbf{I}$ and the DoS rule in Case II are used in turn. The one that enables a larger trade amount will be finally adopted.

\section{Features of GDAM}

Double auctions have some features, which can also be evidenced in our mechanism, such as strategy-proof, weakly budget-balanced, etc.

Theorem 1: Under the assumption that the QoS requirements(prices and quantities) of the buyers and sellers are private informations, GDAM is strategy-proof with respect to both QoS parameters, weakly budgetbalanced and individually rational.

Proof: According to GDAM, the price-quantity broken line of the traded sellers is always below that of the buyers. Assume $M$ and $N$ are the collection of these buyers and sellers respectively, we can get $\sum_{i=1}^{|M|} b r_{i} \cdot p * b r_{i} \cdot q-\sum_{j=1}^{|N|} s r_{j} . p * s r_{j} . q \geq$ 0 which indicates that the mechanism can always get nonnegative payment. Hence our mechanism is weakly budget-balanced. In fact, the nonnegative surplus is supposed to be earned by the market maker for managing the auction market.

As stated in section 3, a bidding price includes the profit expected by the buyer and the asking price contains the profit expected by the seller. Each agent gets expected profit if it succeeds in trading, or zero if it fails. Hence our mechanism is individually rational.

Suppose in the scenario of Case I, a seller $s r_{L}$ over-reports his asking price in order to get more profit, while others remain unchanged(refer to figure 6). In figure 6 if seller $L$ reports his asking price honestly, he will get the surplus, $s s_{L}=\left(s r_{L} \cdot p-s c_{L}\right) s r_{L} \cdot q$ according to (2). If he over-reports his price by an amount of $d$, his surplus becomes $\left(s r_{L} \cdot p+d-s c_{L}\right) s r_{L} \cdot q$ with an extra surplus of $d \cdot s r_{L} . q$. If he continues over-reporting the price by another amount $e$ with $s r_{L} \cdot p+e>b r_{E} \cdot p$, he will lose the opportunity of making successful trade based on SoD rule and gets a surplus of zero. Though over-reporting the price may produce extra surplus, it is hard for a SA to implement this over-reporting strategy successfully under our mechanism. It is easy to explain. Since the QoS requirements of trading agents are transparent between each other, a SA never knows which agents will trade finally, let alone the BA who is "just above" him. Therefore a seller can not decide how much to over-report his price, and the arbitrary over-reporting may actually reduce his surplus as discussed previously. The same proving procedure can be applied to the scenario of Case II where 


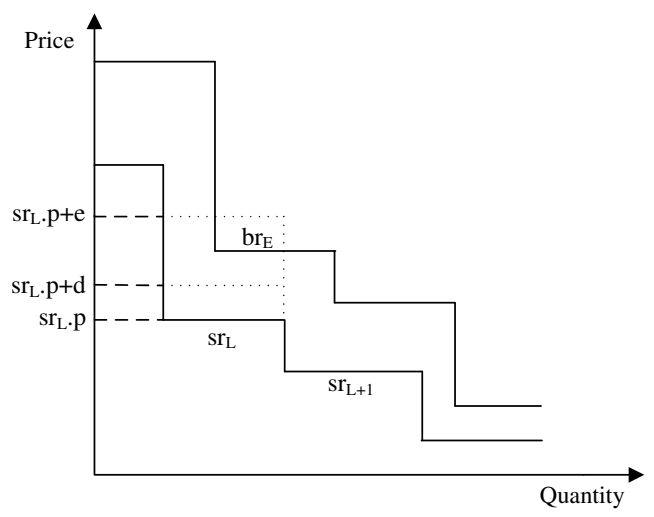

Fig. 6. Seller L over-reports his price while others remain unchanged.

a buyer wants to under-report his price. Since the trading agents have no idea of which auction rule (SoD or DoS) will be adopted by our mechanism and misreporting their prices may take a big risk of losing profit, they will not cheat on their prices. Furthermore, an agent has no incentive to misreport his trading quantity since an agent's trading price will not be affected by the quantity under GDAM. So we can see that GDAM is strategy-proof with respect to both price and quantity.

Theorem 2: GDAM enables larger trading amount than the traditional mechanism, resulting in a better utilizing of grid resources.

Proof: Assume that $K$ buyers and $L$ sellers will finally trade under the traditional auction mechanism. If we arrange these L sellers in the decedent order of price, as illustrated in figure 7, we can see that these L sellers will also succeed in trading according to SoD rule. Moreover, SoD results in a larger trading amount. It works as follows. Seek for the buyer $b r_{E}$, which satisfies $b r_{E}<s r_{1} . p \leq b r_{E-1}$. If $b r_{E}$ does not exist, we move forward to find the buyer $b r_{F}$, which satisfy $b r_{F}<s r_{2} . p \leq b r_{F-1}$. Continue the process until the proper buyer is found. Without loss of generality, assume $b r_{E}$ is found. Let $g=\sum_{i=1}^{E-1} b r_{i} \cdot q-\sum_{j=1}^{L} s r_{j} . q$. If there is a seller $s r_{H}(H>L)$ that satisfies $s r_{H} \cdot q<g$ and will not cause an up-crossing point (will not let (6) and (7) hold), $s r_{H}$, which is abandoned under traditional mechanism, can also trade according to SoD rule. The analysis for DoS rule can be conducted in the same way by arranging the $K$ buyers in the ascend order of price.

\section{$5 \quad$ Experiments and Analysis}

\subsection{Parameter Definition}

The parameters that will be used are given as follows: 


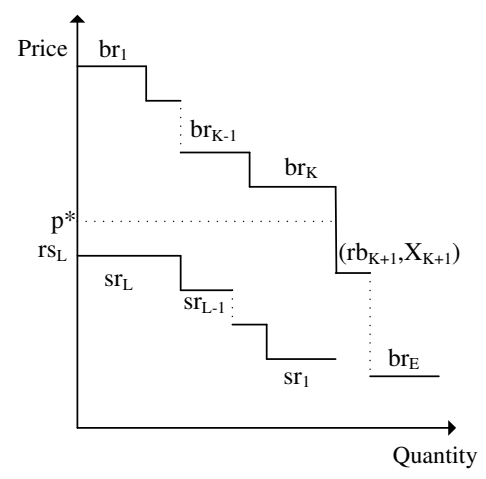

Fig. 7. The L successful sellers are rearranged.

- Number of Agents: We consider two cases. When then number of SA, $n$ equals the number of BA, $m$, we run simulation at $m=n=10,40,70,100$, $200,500,800,1000$ respectively. When $n \neq m$, the simulation is performed at $(m, n)=(20,180),(40,160),(60,140),(80,120),(100,100),(120,80),(140,60)$, $(160,40),(180,20)$ respectively.

- Resource Quantity : We assume that both the resource quantities demanded by buyers and that supplied by sellers are uniformly distributed between 10 and 100 (the unit depends on the kind of resource).

- Resource Price: We assume the sellers' asking prices are uniformly distributed between 50 and 100 (Grid $\$$ ) and the buyers' bidding prices are subject to uniform distribution $U(25,75), U(50,100)$ and $U(75,125)$ respectively.

- Expected Profit: As for seller $j$, his expected profit, $s e p_{j}$, from per unit of resource equals the result of subtracting the resource cost from his asking price. As for buyer $i$, if we subtract his bidding price from the value that he can create from consuming per unit of the resource, we get his expected profit, $b e p_{i}$.

- Aggregate Surplus as: Assume $M$ and $N$ represent the collection of buyers and sellers that can trade respectively. Then the aggregate surplus of the auction market is $\sum_{i=1}^{|M|} b e p_{i} \cdot b r_{i} \cdot q+\sum_{j=1}^{|N|} s e p_{j} \cdot s r_{j} \cdot q$.

- Market Surplus ms: The market surplus is the profit that the market can obtain. Assume $M$ and $N$ represent the collection of buyers and sellers that can trade respectively. Let $v p$ be the total value paid by all the buyers, $v a$ be the total value got by all the sellers. We have $v a=\sum_{i=1}^{|M|} b r_{i} \cdot p \cdot b r_{i} . q$, $v p=\sum_{j=1}^{|N|} s r_{j} \cdot p \cdot s r_{j} \cdot q$ and $m s=v a-v p$.

\subsection{Performance Metric}

The following performance metrics have been used for evaluation. 
- Economic Efficiency Loss eel: In the economic field, a market's efficiency is usually measured by comparing the aggregate profit made by the participants to the maximum profit that theoretically could be made. So under GDAM, the efficiency of the double auction market equals $\frac{a s}{a s+m s}$. We treat the profit obtained by the market maker, $m s$ as an economic loss and the economic efficiency loss can be expressed as eel $=\frac{m s}{a s+m s}$, then the market's economic efficiency is equal to $1-e e l$.

- Trade Amount $t a$ : The trade amount, $t a$ is the total quantity of resources that can be successfully traded under our mechanism. A lager trade amount means a greater utilization of grid resources enabled by the market.

- User Satisfaction Percentage: Under GDAM either a seller or a buyer is the user of the trading service provided by the auction market. The sellers' satisfaction percentage, ssp of the trading service is measured by comparing the number of sellers who trade to the number of all the sellers who participate the auction. The same meaning holds true for the buyers satisfaction percentage, $b s p$.

\subsection{Experiment Procedures}

The first experiment was performed to compare GDAM with the traditional auction mechanism(TAM), actually the one proposed in [13]. The number of the buyers, $m$ is equivalent to the number of the sellers, $n$ and the bidding prices' distribution varies from $U(25,75)$ to $U(75,125)$ while the asking prices' distribution is fixed at $U(50,100)$. The experiment result is shown in table 1 , where $S N$ and $B N$ represent the number of the sellers and buyers that can trade respectively. All the experiment results in this paper are the average of 10,000 same experiments.

We can see when the distribution of bidding price changes from $U(25,75)$ to $U(75,125)$, under each of the three mechanisms both the trade amount and the number of successful agents increase. This is easy to understand for a BA with a higher bidding price has a greater probability to trade. When $b r \cdot p=U(25,75)$ with the same $m$, the trade amount and the number of successful agents produced by TAM are much less compared to SoD. The trade amount made by TAM only takes up an average of $56.1 \%$ of the amount produced by SoD (with a minimum of $53 \%$ and a maximum of $61 \%$ ). This accords with Theorem 2. And SoD satisfies much more users (with the average of $s s p$ and $b s p$ being $40 \%$ and $44 \%$ respectively) compared to TAM (with the average of $s s p$ and $b s p$ being $23 \%$ and $24 \%$ respectively). When $s r . p=U(75,125)$ we can draw a similar conclusion and the user satisfaction percentage is nearly $100 \%$. We can also find that there is merely a slight difference between the results under SoD and DoS in terms of trade amount and user satisfaction percentage, so we can randomly select one out of the two auction rules in Case III for simplicity.

In the second experiment we use the scenario where $n>m$ to simulate Case I and $m>n$ to simulate Case II. The adopted (m,n) pairs are $(20,180)$, $(40,160),(60,140),(80,120),(100,100),(120,80),(140,60),(160,40),(180,20)$ and we assume the bidding price has a distribution of $U(25,75)$. When $m-n<0$ 
Table 1. The auction results under different mechanisms.

\begin{tabular}{|l|l|l|l|l|l|l|l|}
\hline & & \multicolumn{3}{|c|}{$b r \cdot p=U(25,75)$} & \multicolumn{3}{c|}{ br.p $=U(75,125)$} \\
\hline & $n=m$ & $t a$ & $S N$ & $B N$ & $t a$ & $S N$ & $B N$ \\
\hline TAM & 10 & 64.1 & 2 & 2 & 325.1 & 6 & 8 \\
\hline SoD & 10 & 114.5 & 3 & 5 & 452.3 & 8 & 9 \\
\hline DoS & 10 & 121.9 & 3 & 5 & 466.1 & 9 & 9 \\
\hline TAM & 40 & 479.2 & 9 & 11 & 1578.5 & 32 & 36 \\
\hline SoD & 40 & 783.1 & 15 & 16 & 2076.6 & 37 & 39 \\
\hline DoS & 40 & 800.1 & 15 & 17 & 2090.4 & 38 & 39 \\
\hline TAM & 70 & 892.9 & 16 & 17 & 2817.3 & 55 & 47 \\
\hline SoD & 70 & 1515 & 24 & 25 & 3693.2 & 68 & 60 \\
\hline DoS & 70 & 1533.7 & 24 & 26 & 3728 & 69 & 63 \\
\hline TAM & 100 & 1302.6 & 21 & 22 & 4052.9 & 68 & 63 \\
\hline SoD & 100 & 2263.9 & 42 & 41 & 5320.6 & 98 & 92 \\
\hline DoS & 100 & 2284.8 & 42 & 44 & 5335.7 & 99 & 90 \\
\hline TAM & 200 & 2680.7 & 40 & 43 & 8178.1 & 144 & 158 \\
\hline SoD & 200 & 4827.5 & 79 & 83 & 10760.3 & 189 & 199 \\
\hline DoS & 200 & 4849.4 & 75 & 84 & 10760.2 & 189 & 199 \\
\hline TAM & 500 & 6802.4 & 124 & 128 & 20561.3 & 382 & 377 \\
\hline SoD & 500 & 12700.7 & 232 & 234 & 27151 & 496 & 494 \\
\hline DoS & 500 & 12725.6 & 229 & 235 & 27187.1 & 499 & 495 \\
\hline TAM & 800 & 10926.2 & 184 & 200 & 32915.7 & 576 & 590 \\
\hline SoD & 800 & 20692.8 & 352 & 375 & 43549.5 & 767 & 798 \\
\hline DoS & 800 & 20718.7 & 345 & 376 & 43565.5 & 774 & 796 \\
\hline TAM & 1000 & 13673.1 & 267 & 261 & 41169.5 & 751 & 758 \\
\hline SoD & 1000 & 26024.7 & 493 & 479 & 54498.8 & 996 & 993 \\
\hline DoS & 1000 & 26151.4 & 487 & 484 & 54514.9 & 998 & 990 \\
\hline
\end{tabular}

the SoD rule is used to decide which agents will trade; when $m-n>0$ the DoS rule is adopted. The experiment result is illustrated in figure 8 . We can see that with the value of $m-n$ increasing from -160 to 0 the trade amount increases under either mechanism. The trade amount of SoD is always above the trade amount of TAM and this distance becomes larger when $m$ approaches $n$ little by little. When $m=n$ both SoD and DoS produce the maximum trade amount (2258.9 and 2289 respectively). When $m>n$ DoS is adopted and as the value of $m-n$ increases from 0 to 160, the trade amount decreases from 2289 to 514.7. Even the demand quantity is not equivalent to the supply quantity, GDAM still have an advantage over TAM in improving the utilization of grid resources and when this equivalence becomes smaller the advantage becomes more obvious.

We have also performed an experiment to study the economic efficiency of GDAM. Assume $M$ and $N$ represent the collection of buyers and sellers that can trade respectively. Then the market's economic efficiency loss, eel $=\frac{m s}{a s+m s}=$ $\frac{\sum_{i=1}^{|M|} b r_{i} \cdot p \cdot b r_{i} \cdot q-\sum_{j=1}^{|N|} s r_{j} \cdot p \cdot s r_{j} \cdot q}{\sum_{i=1}^{|M|} b e p_{i} \cdot b r_{i} \cdot q+b r_{i} \cdot p \cdot b r_{i} \cdot q+\sum_{j=1}^{|N|} s e p_{j} \cdot b r_{j} \cdot q-s r_{j} \cdot p \cdot s r_{j} \cdot q}$. For simplicity, we assume the 


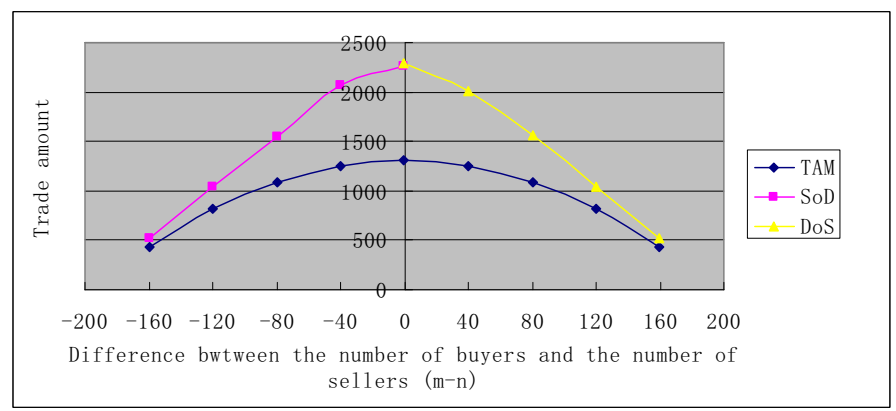

Fig. 8. The auction results with the varying difference between $m$ and $n$.

seller's expected profit equals the buyers', and we assume the expected profit, $e p$ is $0.2,0.4$ and 0.6 respectively. The value of economic efficiency of GDMA (1-eel) is shown in figure 9 . We can see from figure 9 that as the number of the

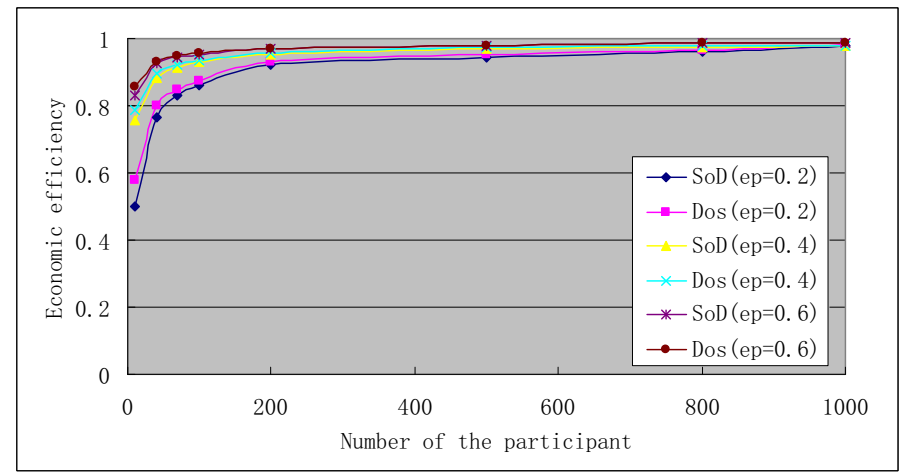

Fig. 9. The economic efficiency of SoD and DoS with $m=n$ and br.p $=U(50,100)$.

participants becomes larger the economic efficiency of our mechanism approaches $100 \%$ nearer. So GDAM is suitable for the environment where there are a huge number of participants, which can well meet the requirement of the grid. Note that as $e p$ increases from 0.2 to 0.6 the corresponding economic efficiency of both SoD and DoS also increases. This is because successful agents with a larger $e p$ get more surpluses, making the economic efficiency loss relatively smaller.

\section{Conclusion}

Traditional double auction mechanisms aim at maximizing the total market value instead of enabling more participants to benefit from the market, which is to 
some extent a kind of system-centric allocation mechanism. In this paper, a novel greedy double auction mechanism(GDAM) is proposed, under which both SAs and BAs consume the trading service that can meet their own QoS requirements of the expected price and amount. What is more important, to satisfy as more participants as possible, GDAM makes use of SoD and DoS rules in different cases. The advantages of GDAM have been proved, such as strategy-proof and individual rational. Simulation results also confirm that GDAM outperforms the traditional one on both the total trade amount and the user satisfaction percentage. And as more agents join in the auction market, the economic efficiency of GDAM will also increase making it is very suitable for the grid environment.

\section{References}

1. Buyya, R., Abramson, D., Giddy, J.: A case for economy grid architecture for service oriented grid computing. In: IPDPS '01: Proceedings of the 15th International Parallel \& Distributed Processing Symposium, Washington, DC, USA, IEEE Computer Society (2001) 83

2. Rajkumar Buyya, D.A., Venugopal, S.: The grid economy. In: Proceedings of the IEEE, IEEE Computer Society (2005) 698-714

3. Sutherland, I.E.: A futures market in computer time. Communications of the ACM 11(6) (1968) 449-451

4. Buyya, R.: Economic-based distributed resource management and scheduling for grid computing. PhD thesis, Monash University, Melbourne, Australia (2002)

5. Regev, O., Nisan, N.: The popcorn market - an online markets for computational resources. In: ICE '98: Proceedings of the first international conference on Information and computation economies, ACM (1998) 148-157

6. Waldspurger, C.A., Hogg, T., Huberman, B.A., Kephart, J.O., Stornetta, S.W.: Spawn: A distributed computational economy. IEEE Transactions on Software Engineering 18(2) (1992) 103-117

7. Bubendorfer, K.: Fine grained resource reservation in open grid economies. In: E-SCIENCE '06: Proceedings of the Second IEEE International Conference on e-Science and Grid Computing, IEEE Computer Society (2006) 81

8. Lalis, S., Karipidis, A.: Jaws: An open market-based framework for distributed computing over the internet. In: GRID '00: Proceedings of the First IEEE/ACM International Workshop on Grid Computing, London, UK, Springer-Verlag (2000) $36-46$

9. Lai, K., Rasmusson, L., Adar, E., Sorkin, S., Zhang, L., Huberman, B.A.: Tycoon: an implemention of a distributed market-based resource allocation system. Technical report, HP Labs (2004)

10. Satterthwaite, M.A., Williams, S.R.: The rate of convergence to efficiency in the buyer's bid double auction as the market becomes large. The Review of Economic Studies 54(6) (1989) 477-498

11. Williams, S.R.: Existence and convergence of equilibria in the buyer's bid double auction. The Review of Economic Studies 58(2) (1991) 351-374

12. Yokoo, M., Sakurai, Y., Matsubara, S.: Robust double auction protocol against false-name bids. In: ICDCS '01: Proceedings of the The 21st International Conference on Distributed Computing Systems, Washington, DC, USA, IEEE Computer Society (2001) 137-145 
13. Huang, P., Scheller-wolf, A., Sycara, K.: Design of a multiunit double auction e-market. Computational Intelligence 18(4) (2002) 596-617

14. Kant, U., Grosu, D.: Double auction protocols for resource allocation in grids. In: ITCC '05: Proceedings of the International Conference on Information Technology: Coding and Computing (ITCC'05) - Volume I, Washington, DC, USA, IEEE Computer Society (2005) 366-371

15. Liu, Y., He, H.C.: Multi-unit combinatorial auction based grid resource coallocation approach. In: SKG '07: Proceedings of the Third International Conference on Semantics, Knowledge and Grid, Washington, DC, USA, IEEE Computer Society (2007) 290-293

16. Gonen, R., Lehmann, D.: Optimal solutions for multi-unit combinatorial auctions: branch and bound heuristics. In: EC '00: Proceedings of the 2nd ACM conference on Electronic commerce, New York, NY, USA, ACM (2000) 13-20

17. Chien, C.H., Chang, P.H.M., Soo, V.W.: Market-oriented multiple resource scheduling in grid computing environments. In: AINA '05: Proceedings of the 19th International Conference on Advanced Information Networking and Applications, Washington, DC, USA, IEEE Computer Society (2005) 867-872

18. Wieczorek, M., Podlipnig, S., Prodan, R., Fahringer, T.: Applying double auctions for scheduling of workflows on the grid. In: SC '08: Proceedings of the 2008 ACM/IEEE conference on Supercomputing, Piscataway, NJ, USA, IEEE Press (2008) 1-11 